Mots. Les langages du politique

\title{
Le discours politique à l'épreuve de l'essai documentaire (1974, une partie de campagne de Raymond Depardon)
}

Political discourse true-tested in Raymond Depardon's documentary essay

(1974, Une partie de campagne)

El discurso político a prueba del trabajo documental (Raymond Depardon, 1974, une partie de campagne)

Jacques Gerstenkorn

\section{OpenEdition}

Journals

Édition électronique

URL : https://journals.openedition.org/mots/22486

DOI : $10.4000 /$ mots. 22486

ISSN : 1960-6001

Éditeur

ENS Éditions

Édition imprimée

Date de publication : 15 octobre 2016

Pagination : 93-98

ISBN : 978-2-84788-850-8

ISSN : 0243-6450

\section{Référence électronique}

Jacques Gerstenkorn, « Le discours politique à l'épreuve de l'essai documentaire (1974, une partie de campagne de Raymond Depardon) », Mots. Les langages du politique [En ligne], 112 | 2016, mis en ligne le 15 octobre 2018, consulté le 23 avril 2022. URL : http://journals.openedition.org/mots/22486 ; DOI : https://doi.org/10.4000/mots.22486 


\section{Le discours politique à l'épreuve de l'essai documentaire (1974, une partie de campagne de Raymond Depardon)}

On fait souvent de Raymond Depardon le champion français du cinéma direct: le cinéaste serait tel « une mouche collée contre un mur», selon le mot fameux de Richard Leacock, pionnier du cinéma direct américain. Sa position d’observateur lui interdirait d'intervenir de quelque manière dans la situation enregistrée par la caméra et seule une telle posture, qui proscrit l'entretien, la mise en scène et le commentaire en voix off, au même titre que l'ajout d'une musique ou encore les artifices de montage, serait à même de garantir la neutralité et l'impartialité du regard du filmeur. Au dos de la jaquette du DVD de 1974, une partie de campagne, édité par Arte Vidéo, le film est présenté comme «un document essentiel sur la conquête du pouvoir». Un «document» ? Cela n'est assurément pas si simple et nous nous efforcerons ici de montrer que Raymond Depardon, lorsqu'il filme les scènes de la vie politique, met en place un point de vue tout à la fois personnel et réflexif. L'effet de transparence induit par le parti pris de filmage n'est qu'une grande illusion : pour peu que l'on y prenne garde, Depardon porte sur les hommes politiques un regard qui se démarque résolument des images produites par les médias. La partition documentaire proposée par le cinéaste procède d'une interprétation de la réalité filmée, elle offre un contrechamp audiovisuel aux représentations médiatiques et se voudrait sans doute leur antidote. C'est là même, à notre sens, le principal enjeu de l'approche proposée par Raymond Depardon, en dépit des apparences, pour le coup fort trompeuses, et des idées reçues sur la tradition documentaire dont il est l'héritier. Un documentaire n'est pas qu'un document, c'est une élaboration complexe où la subjectivité de l'observateur le dispute aux multiples choix techniques et artistiques engagés par la fabrication d'un film, qui plus est un film d'auteur.

Le premier long métrage documentaire de Raymond Depardon, 1974, une partie de campagne, dont le titre originel était 50,81\%, participe de part en part d'un parti pris radical : rendre compte d'une campagne électorale présidentielle 
en s'attachant à ne suivre qu'un seul candidat, en l'occurrence Valéry Giscard d'Estaing, à tel point qu'à l'issue de la projection, un spectateurvierge de toute culture politique aurait presque du mal à reconnaître le principal adversaire du moment. C'est peu dire que François Mitterrand n'y fait que de la figuration, il n'est pour R. Depardon qu'une silhouette entraperçue juste avant que ne commence le grand débat télévisé entre les deux tours de scrutin. À cette focalisation sur Giscard s'ajoute alors un autre parti pris du cinéaste, encore plus audacieux et paradoxal, qui est d'évacuer le plus possible du film la parole publique du candidat, qu'il s'agisse des discours prononcés à l'occasion des nombreuses réunions électorales ou de ses prises de parole lors des émissions de télévision dédiées à la campagne. Bien malin là encore qui pourrait restituer, en sortant du cinéma, la tenue du programme que Valéry Giscard d'Estaing propose aux Français, un programme qu'on pourrait résumer, si le film était la seule source d'information sur cette campagne électorale, de la manière suivante: «Je suis quelqu'un de sympathique, faites-moi confiance ». À s'en tenir à ce que nous montre R. Depardon, il est impossible d'avoir la moindre idée de la situation de la France et des préoccupations des Français en cette année 1974 ! Si l'on devait soutenir l'idée que, sous la Cinquième République, l'élection présidentielle se fait, conformément au vœu du Général de Gaulle, par «la rencontre d'un peuple et d'un homme », le film de Raymond Depardon en proposerait la plus éclatante confirmation.

Les stratégies déployées par le cinéaste pour reléguer au second plan le discours politique sont multiples, à commencer par la scène d'ouverture en forêt qui met en scène la rêverie d'un promeneur solitaire avec son chien et qui est complètement silencieuse... On conviendra qu'une introduction aussi champêtre à une campagne électorale est pour le moins inattendue, à moins de prendre à la lettre l'expression «partie de campagne», expression au double sens malicieux qui est devenue en 2002 le titre définitif du film, lors de sa sortie juste avant la présidentielle. D’une manière plus générale, il convient de souligner que ce ne sont pas les réunions électorales en tant que telles qui constituent le fil rouge de cette chronique de campagne, pas davantage que les rouages de son organisation même, mais bien les déplacements du candidat. En voiture, en avion, en hélicoptère, en train ou encore remontant les rues à pied, la caméra de Depardon ne quitte pas le candidat d'une semelle. Les croquis abondent : Giscard s'accordant un moment de récupération au cours d'un vol, se recoiffant d'un coup de peigne furtif en voiture (il est déjà bien dégarni mais toujours coquet), commentant la presse, s’inquiétant du déroulement de la campagne, parcourant ses notes pour le prochain discours, échangeant avec sa garde rapprochée (Depardon saisit au passage à plusieurs reprises sa complicité avec Michel Poniatowski, son éminence grise appelée à devenir ministre de l'Intérieur). Fait plus inattendu dans un contexte français, Giscard est fréquemment entouré de ses proches, de son épouse ou ses filles 
(«les deux atouts de charme de la campagne», peut-on lire dans un article de journal), toutes trois embarquées dans une campagne à l'américaine, à la Kennedy (elles partagèrent également à l'époque les affiches électorales du candidat...). Les saynètes d'accueil se répètent, la parade folklorique comme rituel de bienvenue, la remise des bouquets de fleurs, les serrages de mains, la signature d'autographes et les bises aux petites filles pour les photographes. Scènes ordinaires de la vie de campagne, dira-t-on, de toute campagne électorale, mais le charme du film doit beaucoup à cette proximité avec un candidat d'autant plus conscient de la présence de la caméra à ses côtés qu'il est, ne l'oublions pas, le commanditaire du film, comme s'il s'agissait alors pour lui de fixer pour la postérité son pouvoir de séduction sur les foules («Vous avez filmé la foule?», demande ingénument Giscard au cinéaste juste après le grand meeting de la porte de Versailles). L'image est ici plus parlante que mille discours : Giscard prend un plaisir manifeste à fendre la foule et à partir à la rencontre des Français (il se présente alors comme «le président de tous les Français »), il goûte ce moment avec une gourmandise qui n'échappe pas à l'objectif de Depardon. L'essentiel de cette chronique de campagne est là, dans ces gestes qui échappent à l'œil des médias et qu'on pourrait aussi bien appeler le «hors-champ médiatique».

Cette focalisation sur l'homme - le film est à cet égard bien plus un portrait qu'une chronique - est autant un choix de tournage qu'un fait de montage. Quoi de plus de plus répétitif et fastidieux que de filmer une réunion électorale? Un cinéaste moins inspiré se serait contenté de capter le discours de Giscard et l'aurait agrémenté de plans de coupe sur l'assistance. En la circonstance, Depardon adopte pour sa part le parti inverse : il choisit à chaque fois de squeezer le propos de l'orateur, n'en donnant au mieux à entendre que quelques bribes lénifiantes. Filmé tantôt en plan large, tantôt en plans plus serrés sur tel ou tel groupe de militants, c'est bien le public de ces meetings, comme ces jeunes giscardiens qui arboraient sur leur tee-shirt un slogan mémorable ("Giscard à la barre»), et non le discours du candidat, qui retient l'attention de la caméra et par voie de conséquence du spectateur du film. Aussi Depardon se tient-il volontiers derrière Giscard, le prenant de dos face au public, ce qui correspond très précisément au contrechamp de l'angle ordinairement adopté par les caméras de télévision. Surtout, Depardon privilégie au montage les moments qui précèdent l'arrivée du candidat. Il prend soin de nous montrer les chanteurs qui «chauffent» la salle, parmi lesquels on reconnaît cette année-là Dany et Aznavour - ce dernier étant inaudible du fait de la clameur de la salle à l'arrivée de Giscard, recouvrement sonore plutôt moqueur dont on ne sait s'il résulte d'un montage ou bien d'une prise d'ambiance directe... Autant qu'à l'entrée en scène du candidat, Depardon s'attache, à l'issue du meeting, à filmer son bain de foule rituel au cours duquel, selon les propres mots de Giscard, le public est invité à lui souhaiter «bonne chance». Autre rite de clôture, 
le fait de proposer au public d'entonner le chant du Départ, dont Giscard a fait son hymne de campagne ${ }^{1}$. Enfin, tout comme il le fera quelques années plus tard dans Reporters, Depardon prête une attention toute particulière au travail de ses collègues journalistes, comme lorsqu'il balaie, d'un ample mouvement de caméra, les magnétophones de la presse radiophonique présente au meeting de la Porte de Versailles (rien n'est plus daté que la modernité technologique des moyens de communication propres à une époque).

Cette prise de distance vis-à-vis du discours politique se retrouve quand il s'agit de rendre compte des prestations télévisuelles. Ainsi nous ne verrons du grand débat télévisé de l'entre-deux tours que ses coulisses, avec l'arrivée et l'accueil de Valéry Giscard d'Estaing dans l'arène du studio. Dès que l'émission proprement dite commence, la caméra de $R$. Depardon se retire, en même temps que les photographes. Comment ne pas voir dans ce retrait même la métaphore du dispositif adopté par le cinéaste tout au long de ce que Giscard lui-même appelle son «film de la campagne»? Quant aux émissions réalisées dans le cadre de la campagne télévisuelle, Depardon en saisit non pas l'enregistrement mais la diffusion, au cours de repas assez mondains où les convives regardent la télévision et comparent en direct les prestations respectives de Jacques Chaban-Delmas (jugé très mauvais) et de Giscard (couvert d'éloges). Choix ô combien judicieux puisqu'on se souvient de l'effet désastreux produit par les apparitions télévisuelles de «Chaban», alors qu'à l'inverse l'aisance croissante de Giscard lui permettait de grimper dans les intentions de vote. On pourrait en conclure que Depardon cultive tout au long d'Une partie de campagne ce qu'on a pu appeler «l'effet pull-over»: la campagne électorale paraît se jouer davantage sur l'image du candidat que sur le contenu de son programme politique, le téléspectateur conservant en mémoire la couleur de la cravate plutôt que les propos du candidat. De fait, face à ses lieutenants, entre les deux tours, Giscard se lance dans une tirade mémorable où il semble théoriser cette posture qui consiste «à en dire le moins possible pour ne fâcher personne». Ce moment de réflexivité saisi par Depardon fait aussi le prix de la démarche d'ensemble du cinéaste : donner à entendre le «off » de la campagne, cultiver le plus possible l'empathie et la proximité avec l'homme Giscard d'Estaing plutôt qu'avec le candidat en représentation médiatique.

Quelques années plus tard, dans Reporters, Raymond Depardon adoptera une stratégie très similaire pour filmer les personnalités politiques les plus en vue du moment. Nous sommes alors en période de précampagne, en octobre 1980, à près de six mois de l'élection présidentielle. Depardon accompagne Jacques Chirac dans une tournée des boutiques de la rue Saint-Dominique, ce qui donne lieu à une séquence d'anthologie où l'on ne sait si l'on doit admirer le sens du contact du maire de Paris, son énergie et sa bonne humeur (alors

1. Sur le rôle de ce chant dans le film et dans la campagne, voir Goutte, 2012. 
même qu'il avait à l'époque une image médiatique bien moins sympathique) ou si l'on doit à l'inverse s'offusquer de la comédie politique ainsi mise à nu. Un peu plus loin, dans le même film, nous aurons droit à un numéro de haute volée de Mitterrand prenant la parole, dans un salon du Sénat, devant un parterre d'intellectuels. Puis nous assisterons en direct au fiasco que fut l'annonce, par Michel Rocard, de sa volonté d'être candidat à la présidence de la République, déclaration mise en scène devant la mairie de Conflans-SainteHonorine. Pour chacune de ses séquences, la caméra capte la théâtralité du jeu politique et, par là-même, fait tomber les masques². Le cinéma direct se révèle ainsi un formidable outil d'analyse des stratégies médiatiques pratiquées par les hommes politiques, à commencer par les candidats (déclarés ou non) à la présidence de la Républiques.

\section{Références}

Comolu Jean-Louis, SAmson Michel, 2005 [1994], La campagne de Provence. Chronique d'une élection, J.-L. Comolli, M. Samson, Marseille contre Marseille, 1, Paris, Doriane Films, 2 DVD vidéo, $6 \mathrm{~h} 1 \mathrm{~min}$.

Cuau Bernard, 2006 [1980], Paroles d'homme, s. l., 1 DVD vidéo, 56 min.

Goutte Martin, 2012, «50,81 \% (Depardon, 1974). La victoire en déchantant», J. Gerstenkorn, M. Goutte éd., Cinémas en campagne. De la chronique électorale à la fiction politique, Lyon, Fage, p. 22-24.

Jeuland Yves, 2011, Le Président, Paris, France Télévisions, 2 DVD vidéo, 4 h 50 min.

Le PaIG E Hugues, VoIsy Yan, 2011, Le Prince et son image, Bois-Colombes, Les Films du paradoxe, 1 DVD vidéo, 1 h 33 min.

2. De ce point de vue, le prix d'interprétation pourrait être attribué, à titre posthume, à Georges Frêche, saisi par la caméra en flagrant délit de fabulation autobiographique dans Le Président (Jeuland, 2010).

3. Usant d'un mode opératoire très différent, Hugues Le Paige a proposé, dans Le Prince et son image (2011), un retour passionnant et lucide sur son expérience de «biographe audiovisuel» de François Mitterrand. Par ailleurs, s'agissant de l'analyse audiovisuelle du discours de campagne, on pourra revoir avec profit La campagne de Provence de J.-L. Comolli et M. Samson (2005), tourné à l'occasion des élections régionales en région Provence-Alpes-Côte-d'Azur, ainsi que Paroles d'homme (Cuau, 1980). 
